\title{
LA MÚSICA COMO HERRAMIENTA FACILITADORA DEL APRENDIZAJE DEL INGLÉS COMO LENGUA EXTRANJERA
}

\author{
Music as a facilitating tool of learning English as a foreign \\ language
}

\section{La musique comme outil facilitateur de l'apprentissage de l'anglais comme langue étrangère}

Carmen María Toscano-Fuentes* y María Carmen FonseCa MorA**

Universidad de Huelva. Departamento de Filología Inglesa. Pabellón, 11 Alto, 39. Avda. Tres de Marzo, s/n. 21071 Huelva (España). Correo-e: *carmen.toscano@ dfing.ubu.es $y^{* * *}$ fonseca@ubu.es

Fecha de recepción: marzo de 2012

Fecha de aceptación definitiva: julio de 2012

Biblid [(1130-3743) 24, 2-2012, 197-213]

RESUMEN

Numerosas investigaciones aseguran que la inclusión de canciones y/o de elementos sonoro-musicales en la enseñanza de lenguas extranjeras (LE) aporta beneficios en el proceso de aprendizaje a niveles lingüísticos, afectivos y sociolingüísticos. El objetivo central de este estudio fue el de mostrar los resultados de un programa sonoro-musical puesto en práctica en la enseñanza de inglés como LE con escolares españoles de sexto de primaria. Primeramente, se estudió cuantitativamente si el alumnado con habilidades auditivas avanzadas aprendía una LE de forma más efectiva y si éstos mostraban también un alto nivel de desarrollo de la inteligencia musical. Seguidamente, se puso en marcha la intervención sonoro-musical para comprobar si aquellos estudiantes con un bajo nivel de inteligencia musical podrían mejorar su capacidad auditiva y su competencia comunicativa. Los resultados apuntan que 
LA MÚSICA COMO HERRAMIENTA FACILITADORA DEL APRENDIZAJE DEL INGLÉS COMO LENGUA EXTRANJERA

este programa no solo incrementa la comprensión oral, sino la producción oral, la lectora y la motivación del alumnado.

Palabras clave: programa sonoro-musical, aprendizaje de lengua extranjera, música, aptitud lingüística, aptitud musical, enseñanza de inglés.

\section{SUMMARY}

A great number of researches ensure that the inclusion of songs and/or soundmusic elements in the teaching of foreign languages provides benefits in the learning process at linguistic, affective and sociolinguistic levels. The main objective of this study was to show the results of a sound-music program of English teaching with Spanish sixth grade students. Firstly, a quantitative analysis was carried out to check if students with good auditory ability learned a foreign language more effectively, and, they showed a well-developed musical intelligence. After that, a sound-music program of English was led to verify if those students with low level in musical intelligence could improve their listening ability and their communicative competence. The results of this study suggest that this program improves not only the listening comprehension skill but speaking, reading and learners' motivation as well.

Key words: sound-music program, foreign language learning, music, language aptitude, musical aptitude, teaching English.

\section{SOMMAIRE}

De nombreuses recherches confirment les effets bénéfiques que l'incorporation de chansons ou d'éléments sonores musicaux en classe de langue étrangère peut avoir sur le processus d'apprentissage des élèves, tant au niveau linguistique que sociolinguistique ou affectif. L'objectif central de cette étude était de montrer les résultats d'un programme sono-musical utilisé pour enseigner l'anglais à des élèves espagnoles de $\sigma^{\text {ème }}$. Une première étude quantitative a permis d'examiner si les élèves présentant des habiletés auditives avancées apprenaient plus efficacement la langue étrangère et s'ils possédaient un niveau élevé d'intelligence musicale. Ensuite, le programme sono-musical a été appliqué afin de vérifier si les élèves présentant un bas niveau d'intelligence musicale amélioraient leur capacité auditive ainsi que leur compétence communicative. Les résultats suggèrent que ce programme améliore non seulement la compétence de compréhension orale des apprenants, mais aussi leurs compétences de compréhension écrite et de production orale, ainsi que leur motivation.

Mots clés: programme sono-musical, apprentissage des langues étrangères, musique, aptitude linguistique, aptitude musicale, enseignement de l'anglais. 


\section{INTRODUCCIÓN}

Numerosas investigaciones aseguran que la inclusión de canciones o elementos sonoros-musicales en la enseñanza de lenguas extranjeras (LE) aporta beneficios en el proceso de aprendizaje. Uno de los mayores problemas al estudiar una LE es la constante y continua carencia de input auditivo en la lengua meta a la que el alumnado está expuesto. Estudios en este línea muestran como, en términos generales, la utilización de canciones facilita la memorización (Schellenberg et al., 2007), la motivación (Falioni, 1993; Murphey, 1990; Crookes y Schmidt, 1991; Fonseca y García, 2010), además de mejorar las cuatro destrezas básicas (Thain, 2010; Toscano-Fuentes, 2011; Kraus y Chandrasekaran, 2010). La incorporación de música instrumental y vocal en las sesiones de LE ayuda tanto al desarrollo de niveles lingüísticos (fonético, fonológico, morfosintáctico, semántico y léxico), de factores afectivos (reducción de ansiedad, aumento de motivación), como sociolingüísticos (exposición a variedades y registros de la lengua). Touriñán y Longueira (2010, 165) proponen una clasificación más exhaustiva desde el punto de vista musical aunque valiosa para la perspectiva del aprendizaje de idiomas extranjeros. Los dividen en cuatro ámbitos: el cognitivo (desarrollo de la memoria y las representaciones mentales; mejora de la atención; aprendizaje de segundas lenguas); el físico y psicomotriz (control de la respiración; potenciación de los mecanismos de relajación); el socioafectivo (mejora de la confianza en uno mismo; progreso en el grado de tolerancia en las relaciones interpersonales; control de las emociones y la expresión de los sentimientos), y el del criterio personal (práctica de la comunicación y de la empatía; escucha a los demás; desarrollo de la sensibilidad y el sentimiento).

Tradicionalmente se ha debatido mucho si el buen aprendiz de idiomas era aquel con un coeficiente intelectual alto o, por el contrario, poseía una serie de cualidades que lo distinguían del resto. Aunque la mayoría de las personas son capaces de aprender una LE hasta un cierto grado de competencia, algunos alumnos están en mejores condiciones para el aprendizaje de un idioma extranjero que otros. Skehan $(1998,201)$ revisó estudios sobre aptitud lingüística y definió esta capacidad humana como un concepto triárquico basado en "la capacidad auditiva, capacidad lingüística y la capacidad de la memoria”. Este último subcomponente, la capacidad de memoria, es de vital importancia para la comprensión del proceso de aprendizaje de idiomas, a pesar de que todavía refleja una perspectiva estrecha de lo que significa hablar una lengua extranjera, ya que no se trata únicamente de recuperar el material verbal basado en el conocimiento sistemático de la lengua, sino también en conectar con el conocimiento contextual, ya que la interacción y su comprensión no son independientes del contexto (Anderson y Lynch, 1988). Skehan examinó las tres habilidades de una muestra de estudiantes de idiomas calificados como excelentes concluyendo que ni el coeficiente intelectual ni la capacidad cognitiva tenían que ser excepcionales $(1998,212)$. Como conclusión general, Skehan (1998) afirmó que los estudiantes excepcionales de lenguas 
extranjeras son los que en un período relativamente corto de tiempo (unos tres años) se comunican con fluidez y propiedad, y poseen una capacidad de memoria verbal muy desarrollada, es decir, estudiantes que asimilan grandes cantidades de materiales verbales nuevos y los recuperan con facilidad mientras que interactúan. Observó también que el alumnado menos aventajado en el aprendizaje de lenguas extranjeras poseía una habilidad auditiva baja, es decir, que presentaba dificultades para discriminar elementos prosódicos propios de la lengua.

Este planteamiento induce a pensar que la incorporación de melodías en las aulas de idiomas sería una buena alternativa para el alumnado más lento en el aprendizaje de una lengua extranjera pues, por un lado, favorecería el desarrollo de los tres subcomponentes de la aptitud lingüística, el aumento de la habilidad auditiva, un reforzamiento de la habilidad lingüística con el estudio de la letra de las canciones, además de facilitar la memorización al fusionarse elementos sonoros con lingüísticos; y, por otro lado, proporcionaría un acercamiento al alumnado "ya que la música es uno de los centros de interés que está presente en todas las etapas de la vida" (Megías y Rodríguez, 2001, tomado de Touriñán y Longueira, 2010, 166). De hecho, distintos estudios ratifican el vínculo existente entre ambas disciplinas, música y lenguaje, por el gran número de similitudes que comparten.

\section{LENGUAJE-MÚSICA: DOS CAPACIDADES HERMANAS}

Lenguaje y música son dos capacidades hermanas por el origen de su evolución (Brown, 2001) y las similitudes encontradas entre ambas (Mithen, 2005; Patel, 2003; Sloboda, 1989), con un punto de unión: la melodía y el ritmo del habla, conectados directamente con la entonación (Fonseca-Mora, Toscano-Fuentes y Wermke, 2011)

Similitudes

1. Son capacidades universales y específicas del ser humano.

2. Poseen tres modos de expresión: vocal, gestual y escrita.

3. Tanto el habla como el canto se desarrollan en los infantes al mismo tiempo de manera espontánea.

4. El medio natural de ambos es el auditivo vocal.

5. La habilidad de crear un número ilimitado de secuencias nuevas usando elementos musicales o palabras.

6. Ambas siguen un orden fijo en la estructura, unas reglas gramaticales, bien mediante palabras o notas musicales creando una melodía o frase.

7. En ambas surgen, en primer lugar, las capacidades receptivas y seguidamente las productivas. 
LA MÚSICA COMO HERRAMIENTA FACILITADORA DEL APRENDIZAJE DEL INGLÉS COMO LENGUA EXTRANJERA

8. Aparecen diferenciaciones con respecto a la forma cultural y al contexto en el que tales formas son presentadas, lo que afecta al entendimiento de la adquisición.

Diferencias

1. Mientras que un idioma puede ser traducido completamente a otro diferente, no tiene sentido traducir la música de una cultura a otra.

2. Las reglas de un estilo musical no proporcionan información semántica de la misma manera que la gramática aporta al lenguaje.

3. Las reglas de los estilos musicales varían a más velocidad que la gramática de un idioma.

4. Mientras que el lenguaje asevera o pregunta acerca del mundo real, los objetos o las relaciones, la música no posee una función manipuladora.

5. La música precisa de menos demanda cognitiva que el lenguaje, pues los oyentes no necesitan inferir necesariamente los pensamientos e intenciones del compositor o el músico.

6. Las expresiones emocionales son una parte central de la música, pero no siempre del lenguaje.

7. Mientras que la estructura temporal y la organización rítmica juegan un papel fundamental tanto en la música como en el lenguaje, la métrica es específica según la pieza musical, y la estructura suprasegmental prosódica del lenguaje es menos específica y más variable.

8. Aunque el número de tonos en la octava es similar en las diferentes culturas, el número de fonemas difiere entre los idiomas.

3. LA MÚSICA INSTRUMENTAL Y VOCAL: UN RECURSO PODEROSO PARA LA ENSEÑANZA DE UNA LENGUA EXTRANJERA

Mucho se ha discutido en la literatura sobre la inclusión de elementos sonoros en el aula de idiomas. Aumento motivacional, memorización de elementos lingüísticos en una LE, mejora en la pronunciación han sido los efectos más buscados por los docentes en las clases de lenguas extranjeras. Pero la utilización de la música, bien sea instrumental o vocal, aporta un espectro mucho más amplio y enriquecedor.

\subsection{Efectos de música instrumental}

El uso prolongado de música instrumental ambiental en el aula de inglés como lengua extranjera, en nuestros estudios clásica o new age, ayuda a los estudiantes a relajarse, a concentrarse y también a reducir el ruido de clase (Toscano-Fuentes, 2011; Fonseca-Mora, 2002a). Bancroft (1985) muestra además que provoca cambios en la presión arterial, la respiración, el pulso y la actividad en general. 
Estudios con música de Mozart (Ivanov y Geake, 2003; Hallam, 2000), Bach o Schubert, por citar algunos autores, indican la existencia de una mejora en el razonamiento espacial. Rauscher y Shaw $(1998,839)$ sugieren que aunque con un efecto temporal "complexly structured music, regardless of style or period, may enhance spatial-temporal task performance more readily than repetitious music".

Asimismo, un estructurado entrenamiento musical ayuda a la conciencia fonémica (Overy, 2003; Ehri, Nunes, Willows, Schuster, Yaghoub-Zadeh y Shanahan, 2001; Lonigan, Burgess y Anthony, 2000), y a la discriminación tanto auditiva (Turnipseed, 1976) como rítmica (Ducorneau, 1988; Campbell, 1998) facilitando la mejora de las habilidades lectoras. En un estudio, Fonseca (2002b) resalta también una serie de ventajas: la influencia que ejerce el ritmo de la música sobre el del texto, la identificación y la potenciación de los sentimientos que se producen en él, y el reconocimiento de la macroestructura del fragmento si la música añadida marca las transiciones en el texto escrito.

\subsection{Efectos de la música vocal: las canciones}

El uso de canciones en las aulas de idiomas puede afectar el desarrollo de las cuatro destrezas básicas del aprendizaje de lenguas, es decir, la lectura, la escritura, la audición y el habla (Toscano-Fuentes, 2011; Thain, 2010; Jolly, 1975), además de influir positivamente en la revisión o incorporación de nuevos elementos gramaticales y de vocabulario, "practically all grammar points can be found in music texts, and the texts also offer a wide variety of vocabulary, all of which can be utilized to practice the four communication skills" (Falioni, 1993, 98).

Toscano-Fuentes $(2011,188)$ enfatiza el hecho de que "las canciones activan ambas partes del cerebro debido a que la pronunciación de las palabras, la comprensión, la supervisión de las mismas, el ritmo y la ejecución musical están reservados al hemisferio izquierdo, mientras que la expresión melódica y el timbre que cubre a las palabras, las emociones y la expresión artística (comunicación no verbal) son propios del hemisferio derecho".

Mientras que Campbell $(1992,67)$ asevera que "the more we sing, the more we strengthen the communication between the two sides of the brain", por lo que el aprendizaje se hace más efectivo.

\subsubsection{Efectos que produce escuchar canciones}

Escuchar música es una actividad que en general ayuda a divertirse, a entretenerse, a relajarse o a animarse. Benenzón $(1995,35)$ resume la influencia de la música en el ser humano:

A. Según el ritmo, se incrementa o disminuye la energía muscular.

B. Acelera la respiración o altera su regularidad. 
LA MÚSICA COMO HERRAMIENTA FACILITADORA DEL APRENDIZAJE DEL INGLÉS COMO LENGUA EXTRANJERA

C. Produce un efecto marcado, pero variable, en el pulso, la presión sanguínea y la función endocrina.

D. Disminuye el impacto de los estímulos sensoriales de diferentes modos.

E. Tiende a reducir o demorar la fatiga y, consecuentemente, incrementa el endurecimiento muscular.

F. Aumenta la actividad voluntaria, como escribir a máquina, e incrementa la extensión de los reflejos musculares empleados en escribir o dibujar, por ejemplo.

G. Es capaz de provocar cambios en los trazados eléctricos del organismo.

H. Es capaz de estimular cambios en el metabolismo y en la biosíntesis de variados procesos enzimáticos.

Del mismo modo, el hecho de incluir rima, ritmo y melodías posibilita el aumento de la memorización de la letra (Falioni, 1993), sobre todo cuando la información es significativa (Angelucci et al., 2007; Ho, Cheung y Chan, 2003; Kilgour, Jakobson y Cuddy, 2000). Además, el constante estímulo de fonemas, acentos producidos por la audición de canciones mejora la conciencia fonémica y a su vez la pronunciación (Cardenas-Hagan, Carlson y Durodoloa, 2007; Lightbown y Spada, 2006). Fonseca (2000) aboga por el uso de la melodía musical no sólo para la mejora de la pronunciación, sino de todo el proceso de aprendizaje de la lengua extranjera en general, lo que también mantienen Lems (2005) y Millbower (2000).

\subsubsection{Efectos que produce cantar canciones}

El cantar es una actividad que fusiona tanto la información lingüística como la musical, involucrando a ambas partes del hemisferio a través del cuerpo calloso, el cual se fortalece al transmitir los mensajes entre los hemisferios. La pronunciación, el aumento de vocabulario y la práctica de estructuras gramaticales destacan a este respecto.

Techmeier (1969) asegura que la pronunciación es lo más difícil de adquirir de una lengua ya que hay muchas diferencias entre los elementos fonológicos del inglés y del español que la hacen diferente (Restrepo y Silverman, 2001). En general, las actividades musicales que incluyen las canciones y el ritmo ayudan al desarrollo de la discriminación auditiva (Richards, 2001) y de la pronunciación "there is probably not a better not quicker way to teach phonetics than with songs" (Leith, 1979, 540). Por otro lado, Abbott $(2002,11)$ comenta que "the activity or content of the songs should be related to the theme, topic, or functions that are covered in class. Simple, repetitive songs often contain recurrent grammatical patterns and can be used as grammar practice activities". 
4. PROGRAMa DE INTERVENCIÓN SONORO-MUSICAL EN LA ENSEÑANZA DEL INGLÉS COMO LENGUA EXTRANJERA EN EDUCACIÓN PRIMARIA: LA RELACIÓN ENTRE EL NIVEL DE ADQUISICIÓN DE UNA LENGUA, LA CAPACIDAD AUDITIVA Y LA INTELIGENCIA MUSICAL

\subsection{Objetivo}

El objetivo central de este estudio es el de encontrar respuestas a estos dos interrogantes: ¿Muestran los estudiantes con un alto nivel de desarrollo de la inteligencia musical una mejor capacidad auditiva para el aprendizaje de una LE? ¿Puede un programa sonoro-musical de enseñanza de inglés como lengua extranjera mejorar la capacidad auditiva y, consecuentemente, la aptitud lingüística del alumnado de sexto de primaria?

Debido a esto, se crea un programa de enseñanza del inglés como LE con intervención musical, y con una metodología basada en la enseñanza a través de tareas, con el fin de paliar o subsanar las dificultades halladas en el test de aptitud realizado al alumnado a comienzos de curso. Cada unidad didáctica propuesta al aprendiz incluye la puesta en práctica de una tarea final. No obstante, lo que diferencia a esta metodología de otra es que la música, el input sonoro, se incorpora en todas las sesiones del curso bien a través de canciones, cuentos o simplemente música instrumental de fondo.

En términos generales la música instrumental, mayoritariamente del período barroco y clásico, se escucha constantemente como música de fondo, tanto para las explicaciones como para la realización de las diversas tareas. Del mismo modo, todos los días el alumnado escucha y/o canta una canción proveniente de su libro de texto, del Jazz Chants, del libro de Raps! For learning English, u otras melodías de vocalistas o grupos musicales en lengua inglesa, con el propósito de mejorar la audición, estudiar o repasar vocabulario y/o conceptos gramaticales, centrarse en la pronunciación, entonación, entre otros.

\subsection{Descripción de los participantes}

En este estudio longitudinal han participado 49 alumnos españoles de sexto de primaria de un centro escolar de la localidad de Lepe (Huelva) durante el curso académico 2008-2009. Todos comenzaron a estudiar inglés en 1. ${ }^{\circ}$ de primaria y todos han tenido la misma profesora de idiomas. El colegio está ubicado en el centro de la localidad y cuenta con una población de nivel sociocultural medio.

\subsection{Instrumentos}

Varios han sido los instrumentos que se seleccionaron para la recogida de datos:

- Prueba de nivel de inglés al comienzo y al final del curso académico. 
- $\quad$ Plab (Pimsleur Language Aptitude Battery), pasada al comienzo del curso escolar para comprobar la capacidad auditiva y la aptitud lingüística de cada estudiante.

- Seashore test, que permite conocer la aptitud musical del alumnado.

- Cuestionario de grado de satisfacción de los estudiantes.

- Observación directa del aula realizada por la investigadora.

\subsection{Resultados}

Con el objeto de partir de la verificación de lo que ya se había observado en la revisión de la literatura se estudió la siguiente hipótesis:

Hipótesis 1: el alumnado con habilidades auditivas avanzadas aprende una lengua extranjera de forma más efectiva.

La correlación y regresión lineal simple basada en el test de correlación lineal de Pearson se utiliza para comprobar ambas variables: la capacidad auditiva del alumnado y los niveles de competencia. La significación estadística es 0,000, lo que muestra que hay una relación significativa entre ambas variables ya que los estudiantes con una capacidad auditiva elevada obtienen también mejores resultados en el aprendizaje de la lengua inglesa.

Hipótesis 2: los estudiantes con una buena capacidad auditiva muestran un alto nivel de desarrollo de la inteligencia musical.

Los resultados proceden de la correlación entre los estudiantes que obtuvieron altos niveles de habilidad auditiva según el test 5 y 6 de aptitud de la lengua de Pimsleur y de aquellos con un elevado nivel de inteligencia musical de acuerdo al test musical de Seashore. El análisis muestra una correlación de 0,290, asociado al nivel de significación 0,043, lo que evidencia la relación estadísticamente significativa entre ambas variables, así pues los estudiantes con puntuaciones elevadas en inteligencia musical también poseen altos niveles de capacidad auditiva.

La confirmación de esta hipótesis nos lleva al diseño y puesta en práctica de un programa de enseñanza del inglés a través de tareas con intervención musical con el fin de mejorar, en general, la capacidad auditiva del alumnado de sexto de primaria y, consecuentemente, su competencia comunicativa, si bien desconocíamos en qué medida podría afectar este programa educativo sonoro-musical las demás destrezas lingüísticas.

Hipótesis 3: la incorporación de un programa sonoro-musical afecta el proceso de enseñanza y aprendizaje del inglés como lengua extranjera.

El análisis de los diferentes niveles de competencia inglesa muestra que hay una evolución positiva, especialmente en lo referente a la capacidad auditiva (ver Cuadro 1). 


\section{Cuadro 1. Prueba de muestras Relacionadas}

\begin{tabular}{|c|c|c|c|c|c|c|c|c|c|}
\hline & & \multicolumn{5}{|c|}{ DIFERENCIAS RELACIONADAS } & \multicolumn{3}{|c|}{ PRUEBA DE HIPÓTESIS } \\
\hline & & \multirow[t]{2}{*}{ MEDIA } & \multirow[t]{2}{*}{$\begin{array}{c}\text { DESVIACIÓN } \\
\text { TÍP. }\end{array}$} & \multirow{2}{*}{$\begin{array}{c}\text { ERROR } \\
\text { TÍP. DE } \\
\text { LA MEDIA }\end{array}$} & \multicolumn{2}{|c|}{$\begin{array}{l}\text { 95\% INTERVALO DE } \\
\text { CONFIANZA PARA } \\
\text { LA DIFERENCIA }\end{array}$} & \multirow[t]{2}{*}{$\mathrm{T}$} & \multirow[t]{2}{*}{ GL } & \multirow[t]{2}{*}{$\begin{array}{c}\text { SIG. } \\
\text { (BILATERAL) }\end{array}$} \\
\hline & & & & & INFERIOR & SUPERIOR & & & \\
\hline Par 1 & $\begin{array}{l}\text { Habla Inicial } \\
\text {-Habla Final }\end{array}$ & $-2,1786$ & ,9709 & ,1387 & $-2,4575$ & $-1,8997$ & $-15,707$ & 48 &, 000 \\
\hline Par 2 & $\begin{array}{l}\text { Escritura Inicial } \\
\text {-Escritura Final }\end{array}$ & $-1,1327$ & 1,4305 & ,2044 & $-1,5435$ &,- 7218 & $-5,543$ & 48 &, 000 \\
\hline Par 3 & $\begin{array}{l}\text { Audición Inicial } \\
\text {-Audición Final }\end{array}$ & $-3,6959$ & 1,8085 &, 2584 & $-4,2154$ & $-3,1764$ & $-14,305$ & 48 &, 000 \\
\hline $\operatorname{Par} 4$ & $\begin{array}{l}\text { Lectura Inicial } \\
\text {-Lectura Final }\end{array}$ & $-2,7112$ & 2,2828 &, 3261 & $-3,3669$ & $-2,0555$ & $-8,314$ & 48 &, 000 \\
\hline Par 5 & $\begin{array}{l}\text { Gramática } \\
\text { Inicial-Gramática } \\
\text { Final }\end{array}$ & $-1,8418$ & 1,1811 & ,1687 & $-2,1811$ & $-1,5026$ & $-10,916$ & 48 &, 000 \\
\hline
\end{tabular}

La significación bilateral es, en todos los casos, inferior al criterio alfa 0,05, por lo que nada se opone a rechazar la hipótesis nula. Esto quiere decir que en todos los casos se obtuvieron puntuaciones finales significativamente superiores a las iniciales. Debido a la existencia de diferencias estadísticamente significativas entre la puntuación de las cuatro destrezas tanto al principio y al final del curso académico, se crearon las variables calculadas que se exponen en el Cuadro 2. En cada caso, se trata de la diferencia simple de la puntuación final menos la inicial por lo que una puntuación positiva implica una mejoría y una puntuación negativa significa un peor nivel al final del curso que a su inicio.

La mejora en el habla fue de 2,1786, en la escritura de 1,1327, en la audición de 3,6959, en la lectura de 2,7112, mientras que en la gramática fue de 1,8418. Por tanto, en términos generales, los estudiantes mejoraron su nivel de competencia en la LE con el programa de intervención musical de enseñanza de lenguas extranjeras, siendo las capacidades receptivas, es decir, la audición y la lectura, las que mostraron un mayor nivel de progreso.

Una vez constatado que el alumnado ha incrementado su nivel de competencia en inglés como lengua extranjera, se les pasa un cuestionario de valoración de las sesiones para que mostraran su opinión al respecto. El porcentaje de sus respuestas se resume en el Cuadro 3. 
LA MÚSICA COMO HERRAMIENTA FACILITADORA DEL APRENDIZAJE DEL INGLÉS COMO LENGUA EXTRANJERA

\section{CUADRO 2. ESTADÍSTICOS DESCRIPTIVOS DE LAS PUNTUACIONES DE «EVOLUCIÓN DEL APRENDIZAJE»}

\begin{tabular}{|c|c|c|c|c|c|}
\hline & $\mathrm{N}$ & MÍNIMO & MÁXIMO & MEDIA & DESV. TíP. \\
\hline $\begin{array}{c}\text { Evolución de la } \\
\text { puntuación del } \\
\text { habla (Speaking) }\end{array}$ & 49 & $-1,00$ & 4,00 & 2,1786 &, 97093 \\
\hline $\begin{array}{c}\text { Evolución de la } \\
\text { puntuación de la } \\
\text { escritura (Writing) }\end{array}$ & 49 & $-2,00$ & 4,10 & 1,1327 & 1,43046 \\
\hline $\begin{array}{c}\text { Evolución de la } \\
\text { puntuación de la } \\
\text { audición (Listening) }\end{array}$ & 49 &, 00 & 7,20 & 3,6959 & 1,80854 \\
\hline $\begin{array}{c}\text { Evolución de la } \\
\text { puntuación de la } \\
\text { lectura (Reading) }\end{array}$ & 49 & $-3,60$ & 7,60 & 2,7112 & 2,28278 \\
\hline $\begin{array}{c}\text { Evolución de la } \\
\text { puntuación de la } \\
\text { gramática (Grammar) }\end{array}$ & 49 &,- 50 & 4,50 & 1,8418 & 1,18112 \\
\hline N válido (según lista) & 49 & & & & \\
\hline
\end{tabular}

El análisis de la satisfacción de los estudiantes con el acercamiento musical en el aprendizaje de una lengua extranjera prueba que a la gran mayoría de los encuestados les gusta cantar y trabajar con canciones y música en la asignatura de inglés y están a favor de la inclusión de las canciones en la metodología de aula debido a que observan un beneficio en su futuro aprendizaje. En términos generales les divierte trabajar con las canciones sugeridas por la maestra (98\%) y con música de fondo (94\%). Se sienten más capaces de hablar inglés al final de curso que al comienzo (92\%) y su percepción hacia la asignatura ha variado pues comentan que se sienten más motivados para aprender con esta nueva metodología (94\%). Hay que destacar la pregunta 9 en la que se les cuestionaba si aprender inglés con canciones era un reto para ellos o no, obteniéndose una respuesta bastante pareja. A pesar de la dificultad que entraña el hecho de trabajar con canciones en lengua extranjera, con una pronunciación inglesa o americana, un vocabulario más específico, la mitad de la población encuestada no lo ve como un impedimento o una frustración sino como algo positivo y gratificante.

Otro aspecto a destacar es el cambio de actitud y percepción sobre el trabajo en grupo, pues al comienzo de curso se tuvieron bastantes dificultades con las agrupaciones, pero conforme el curso iba avanzando este hermetismo de trabajar siempre con los mismos compañeros se fue disipando y se consiguió que todos trabajaran con todos, a un $96 \%$ les gusta trabajar con otros estudiantes, bien sean de su clase o de otra. 
LA MÚSICA COMO HERRAMIENTA FACILITADORA DEL APRENDIZAJE DEL INGLÉS COMO LENGUA EXTRANJERA

\section{CuAdro 3. Cuestionario de VAloración de las Clases CON ACTIVIDADES MUSICALES}

\begin{tabular}{|c|c|c|c|}
\hline CANTAR CANCIONES & $\begin{array}{l}\text { 1. TOTALMENTE EN } \\
\text { DESACUERDO \% }\end{array}$ & $\begin{array}{l}\text { 2. PARCIALMENTE } \\
\text { DE ACUERDO } \% \\
\end{array}$ & $\begin{array}{l}\text { 3. TOTALMENTE } \\
\text { DE ACUERDO } \% \\
\end{array}$ \\
\hline 1. Me gusta cantar & 4 & 8 & 88 \\
\hline $\begin{array}{l}\text { 2. Me gusta cantar } \\
\text { canciones en inglés }\end{array}$ & 4 & 0 & 96 \\
\hline $\begin{array}{l}\text { 3. Me gusta cantar en las } \\
\text { clases de inglés }\end{array}$ & 6 & 16 & 78 \\
\hline $\begin{array}{l}\text { 4. Cantar canciones en } \\
\text { inglés mejora mi LE }\end{array}$ & 6 & 8 & 86 \\
\hline $\begin{array}{l}\text { 5. Escucho canciones cantadas } \\
\text { en lengua extranjera en casa }\end{array}$ & 24 & 0 & 76 \\
\hline $\begin{array}{l}\text { 6. Prefiero aprender inglés } \\
\text { con música que con el } \\
\text { método tradicional }\end{array}$ & 2 & 2 & 96 \\
\hline $\begin{array}{c}\text { ACTITUD HACIA EL } \\
\text { APRENDIZAJE DEL INGLÉS } \\
\text { A TRAVÉS DE CANCIONES }\end{array}$ & $\begin{array}{l}\text { 1. TOTALMENTE } \\
\text { EN DESACUERDO }\end{array}$ & 2. REGULAR & $\begin{array}{l}\text { 3. TOTALMENTE } \\
\text { DE ACUERDO }\end{array}$ \\
\hline $\begin{array}{l}\text { 7. Me gusta escuchar las } \\
\text { canciones propuestas por } \\
\text { la maestra de inglés }\end{array}$ & 2 & 4 & 94 \\
\hline $\begin{array}{l}\text { 8. Me divierte aprender } \\
\text { inglés con las canciones }\end{array}$ & 0 & 2 & 98 \\
\hline $\begin{array}{l}\text { 9. El aprendizaje de inglés con las } \\
\text { canciones es un reto para mí }\end{array}$ & 51 & 3 & 46 \\
\hline $\begin{array}{l}\text { 10. Me gusta aprender inglés } \\
\text { más así, que como lo } \\
\text { hacíamos anteriormente }^{1}\end{array}$ & 2 & 2 & 96 \\
\hline $\begin{array}{l}\text { 11. Soy más capaz de hablar } \\
\text { inglés en las clases ahora } \\
\text { que al comienzo del curso }\end{array}$ & 2 & 6 & 92 \\
\hline $\begin{array}{l}\text { 12. Me gusta que la maestra } \\
\text { use música en general } \\
\text { en las clases de inglés }\end{array}$ & 4 & 2 & 94 \\
\hline $\begin{array}{l}\text { 13. Me gusta trabajar con } \\
\text { otros estudiantes }\end{array}$ & 2 & 2 & 96 \\
\hline $\begin{array}{l}\text { 14. Estoy más motivado/a para } \\
\text { aprender inglés después de } \\
\text { las actividades con canciones }\end{array}$ & 2 & 4 & 94 \\
\hline
\end{tabular}

1. Esta pregunta es igual que la número seis del bloque anterior con el objeto de validar esta respuesta. 


\section{CONCLUSIONES}

Se aprecia que ciertas dificultades en el proceso de enseñanza y aprendizaje del inglés como LE en un contexto no bilingüe se han disminuido gracias a la utilización de música y canciones. La inclusión de elementos sonoros se convierte en un instrumento didáctico adecuado y recomendable para las lenguas extranjeras porque favorece la mejora de la pronunciación, las estructuras gramaticales y de las cuatro destrezas básicas.

Si el aprendizaje está íntimamente relacionado con la audición (Pimsleur, 1966), una persona que no sea capaz de oír y decodificar los fonemas de una lengua, independientemente de que sea la materna o una extranjera, será incapaz de memorizarla, recuperarla y ponerla en práctica cuando lo necesite. Esta reflexión basada en los tres elementos integrantes de la aptitud lingüística (Skehan, 1998) pone de manifiesto que para los idiomas es fundamental el equilibrio entre ellos y que la falta de desarrollo en una de las tres habilidades implicaría una dificultad en el aprendizaje de la LE. Las melodías en la enseñanza del inglés como lengua extranjera, de forma global, mejoran la capacidad de aprendizaje de una lengua extranjera del alumnado con una baja habilidad auditiva. Sin embargo, futuros estudios son aún necesarios para analizar el impacto de un programa sonoro-musical en la conciencia fonémica y la fónica de los estudiantes, es decir, la mejora de la capacidad de discriminación de sonidos, la segmentación de las oraciones, la entonación y el ritmo de la lengua, lo que se manifestaría fundamentalmente en la pronunciación y lectura en voz alta del alumnado.

Los datos cualitativos informan que la gran mayoría de los participantes fueron receptivos a este tipo de actividades, tal y como expresaron en los cuestionarios y en las entrevistas individualizadas, así como en los datos proporcionados a partir de la observación directa, enriqueciéndose de diversas maneras:

1. La música de fondo ha facilitado una metamorfosis en sus conductas entre compañeros no sólo a nivel de comportamiento y de respeto hacia la docente.

2. La incorporación de canciones e historias narradas con música aumentaron las situaciones de input oral en LE, observándose lo siguiente:

- Relajación en el alumnado.

- Mejora en el ambiente de clase ya que los roces entre los compañeros, el miedo al error o al qué dirán se redujeron por lo general.

- Aumento de la disposición a comunicarse.

- Aceptación de cada miembro en el grupo-clase.

- Desarrollo de la cohesión grupal.

- Incremento del uso del inglés en el aula de LE por parte del alumnado. 
LA MÚSICA COMO HERRAMIENTA FACILITADORA DEL APRENDIZAJE DEL INGLÉS COMO LENGUA EXTRANJERA

En términos generales, la selección de canciones ha beneficiado a estos estudiantes en el aprendizaje de LE. Todos mejoraron su competencia comunicativa, siendo la comprensión auditiva, la producción oral y la lectura las más favorecidas. Arnold apunta "We learn better if which attracts our attention, and emotion automatically makes us pay attention" $(1999,260)$. Esto es lo que la música proporciona en un programa de aprendizaje de lengua. No consiste sólo en unir elementos prosódicos verbales con estímulos musicales, sino en alcanzar un nivel de significado. La música parece dejar una profunda huella en nuestra memoria ya que apela a factores afectivos de manera inconsciente, lo que llama nuestra atención beneficiando la memoria verbal.

Así, las melodías en la enseñanza de idiomas aportan un medio sonoro más rico, lo que significa que un acercamiento melódico es una alternativa plausible que mejora la conciencia de los sonidos en la enseñanza del inglés como lengua extranjera, los ritmos, la pausa y la entonación. También genera un sentido de comunidad y una relación más colaborativa entre los integrantes del grupo.

Los resultados de esta investigación indican también que el profesorado de LE debería tener a su alcance pruebas de fácil aplicación para conocer las habilidades auditivas, lingüísticas y de memoria verbal de su alumnado, es decir, herramientas de diagnóstico que le permitan plantear programas de intervención educativa adecuados si el estudiante no es capaz de discriminar los sonidos de la nueva lengua o de almacenar el input auditivo que se incorpora en las sesiones, de procesar adecuadamente las actividades gramaticales o si necesita estrategias de aprendizaje basadas en tareas específicas que favorezcan la memoria verbal.

Finalmente, deseamos resaltar que trabajar la inteligencia musical en las clases de lenguas extranjeras puede provocar beneficios tales como la concentración y la conexión con el interior del estudiante, la estimulación del proceso creativo, la reducción de ruidos disuasorios procedentes del exterior, el fomento de una atmósfera de clase relajada, motivadora y productiva. Las actividades relacionadas con la inteligencia musical pueden facilitar el desarrollo más efectivo de la comunicación en el idioma meta ayudando a los aprendices a activar la información lingüística almacenada en su memoria. 


\section{REFERENCIAS BIBLIOGRÁFICAS}

Aввотт, M. (2002) Using Music to Promote L2 Learning among Adult Learners. TESOLJournal, 11 (1), 10-17.

Anderson, A. y Lynch, R. (1988) Listening. Oxford, Oxford University Press.

Angelucci, F.; Fiore, M.; Ricci, E.; Padua, L.; SABino, A. y Tonali, P. A. (2007) Investigating the Neurobiology of Music: Brain-derived Neurotrophic Factor Modulation in the Hippocampus of Young Adult mice. Behaviour of Pharmaceutical, 18 (5-6), 491-496.

Anvari, S. H.; Trainor, L. J.; Woodside, J. y Levy, B. A. (2002) Relations among Musical Skills, Phonological Processing, and Early Reading Ability in Preschool Children. Journal of Experimental Child Psychology, 83, 111-130.

ARNOLD, J. (1999) Affect in Language Learning. Cambridge, Cambridge University Press.

BANCroft, W. J. (1985) Music Therapy and Education. Journal of the Society for Accelerative Learning and Teaching, 10 (1), 3-19.

Benenzon, R. (1995) Manual de musicoterapia. Barcelona, Paidós.

Bolduc, J. y Montésinos-Gelet, I. (2005) Pitch Awareness and Phonological Awareness. Psychomusicology, 19 (1), 3-14.

Brown, J. D. (2001) Using Surveys in Language Programs. Cambridge, Cambridge University Press.

CAmpBell, D. G. (1992) Introduction to the Musical Brain. Saint Louis, MMB Music Inc.

- (1998) El efecto Mozart. Barcelona, Ediciones Urano.

Cardenas-Hagan, E.; Carlson, C. y Durodoloa, S. (2007) The Cross Linguistic Transfer of Early Literacy Skills: The Role of Initial L1 and L2 Skills and Language of Instruction. Language Speech and Hearing Services in Schools, 38 (3), 249-259.

Colwell, C. M. (1988) The Effect of Music on the Reading Readiness Skills of Kindergarten Children. Tallahassee, Florida State University.

Crookes, G. y Schmidt, R. W. (1991) Motivation: Reopening the Research Agenda. Language Learning, 41, 469-512.

Ducorneau, G. (1988) Musicoterapia. La Comunicación Musical: su Función y sus Métodos en Terapia y reeducación. Madrid, EDAF.

Ehri, L. C.; Nunes, S. R.; Willows, D. M.; Schuster, B. V.; Yaghoub-Zadeh, Z. y Shanahan, T. (2001) Phonemic Awareness Instruction Helps Children Learn to Read: Evidence from the National Reading Panel's meta-analysis. Reading Research Quarterly, 36, 250-287.

Falioni, J. W. (1993) Music as Means to Enhance Cultural Awareness and Literacy in the Foreign Language Classroom. Mid-Atlantic Journal of Foreign Language Pedagogy, 7, 97-108.

Fonseca Mora, M. C. (2000) Foreign Language Acquisition and Melody Singing. ELT Journal, 54 (2), 146-152.

- (2002a) Inteligencias Múltiples, Múltiples Formas de Aprender Inglés. Sevilla, Mergablum.

- (2002b) The Role of Musicality of Language in the Acquisition Process of English as a Second Language/El Papel de la Musicalidad del Lenguaje en el Proceso de Adquisición del Inglés como L2 (Tesis doctoral). Ann Arbor, Universidad de Michigan.

Fonseca Mora, M. C. y García Barroso, L. (2010) Aprender Español en Usa: los Medios de Comunicación como Motivación Social. Comunicar, 34, 145-153. 
LA MÚSICA COMO HERRAMIENTA FACILITADORA DEL APRENDIZAJE DEL INGLÉS COMO LENGUA EXTRANJERA

Fonseca-Mora, M. C.; Toscano-Fuentes, C. y Wermke, K. (2011) The relation between Language Aptitude and Musical Intelligence. Anglistik: International Journal of English Studies, 22 (1), 101-118.

Graham, C. (1978) Jazz Chants for children. Oxford, Oxford University Press.

Gromko, J. E. (2005) The Effect of Music Instruction on Phonemic Awareness in Beginning Readers. Journal of Research in Music Education, 53 (3), 199-209.

Hallam, S. (2000) The Effects of Listening to Music on Children's Spatial Task Performance. British Psychological Society Education Review, 25 (2), 22-26.

Herrera, L.; Defior, S. y Lorenzo, O. (2007) Intervención Educativa en Conciencia Fonológica en Niños Prelectores de Lengua Materna española y Tamazight: Comparación de dos Programas de Entrenamiento. Infancia y Aprendizaje, 30 (1), 39-54.

Ho, Y. C.; Cheung, M. C. y Chan, A. (2003) Music Training Improves Verbal but no Visual Memory: Cross-sectional and Longitudinal Explorations in Children. Neuropsychology, 17, 439-450.

Ivanov, V. K. y Geake, J. G. (2003) The Mozart Effect and Primary School Children. Psychology of Music, 31 (4), 405-413.

Johnson, S. y StannetT, K. (2005) Raps! For Learning English. London, Scholastic.

Jolly, Y. (1975) The Use of Songs in Teaching Foreign Languages. Modern Language Journal, 59 (1), 11-14.

Kilgour, A. R.; JaKobson, L. S. y Cuddy, L. L. (2000) Music Training and Rate of Presentation as Mediators of Text and Song Recall. Memory \& Cognition, 28 (5), 700-710.

Kraus, N. y Chandrasekaran, B. (2010) Music Training for the Development of Auditory Skills. Nature Reviews Neuroscience, 11 (8), 599-605.

Leith, W. D. (1979) Advanced French Conversation through Popular Music. The French Review, 52, 537-551.

LEMs, K. (2005) Music Works: Music for Adult English Language Learners. New Directions for Adult E Continuing Education, 107, 13-21.

Lightbown, P. M. y Spada, N. (2006) How languages are learned. Oxford, Oxford University Press.

Lonigan, C. J.; Burgess, S. R. y ANTHOnY, J. L. (2000) Development of Emergent Literacy and Early Reading Skills in Preschool Children: Evidence from a Latent Variable Longitudinal Study. Developmental Psychology, 36, 596-613.

Millbower, L. (2000) Training with a Beat: The Teaching Power of Music. Sterling, VA, Stylus Publishing.

Mithen, S. (2005) The Singing Neanderthals: The Origins of Music, Language, Mind and Body. London, Weidenfeld y Necholson.

MurpheY, T. (1990) The Song Stuck in my Head Phenomenon: a Melodic Din in the LAD? System, 18 (1), 53-64.

OverY, K. (2003) Dyslexia and Music. From Timing Deficits to Musical Intervention. Annals of the New York Academy Science, 999, 497-505.

Patel, A. D. (2003a) Rhythm in Language and Music: Parallels and Differences. Annals of the New York Academy of Sciences, 999, 140-143.

- (2003b) Language, Music, Syntax and the Brain. Nature Neuroscience, 6, 674-681.

Pimsleur, P. (1966a) Testing Foreign Language Learning, en Valdman, A. (ed.) Trends in Language Teaching. New York, McGraw-Hill, 175-214.

- (1966b) Pimsleur Language Aptitude Battery. New York, Harcourt Brace Jovanovich. 
LA MÚSICA COMO HERRAMIENTA FACILITADORA DEL APRENDIZAJE DEL INGLÉS COMO LENGUA EXTRANJERA

Rauscher, F. H. y Shaw, G. L. (1998) Key Components of the Mozart Effect. Perceptual and Motor Skills, 86, 835-841.

Restrepo, L. y Silverman, I. E. (2001) Osmotherapy in acute Stroke: A Call to Arms. Stroke, 32, 811-812.

Richards, J. C. (2001) Curriculum Development in Language Education. Cambridge, Cambridge University Press.

Schellenberg, E. G.; Nakata, T.; Hunter, P. G. y Tamoto, S. (2007) Exposure to Music and Cognitive Performance: Tests of Children and Adults. Psychology of Music, 35, 5-19.

Seashore, C. E.; Lewis, D. y SaEtvit, J. G. (1992) Test de Aptitudes Musicales de Seashore. Madrid, TEA ediciones.

Skehan, P. (1998) A Cognitive Approach to Language Learning. Oxford, Oxford University Press.

Sloboda, J. (1989) The Musical Mind: The Cognitive Psychology of Music. New York, Oxford University Press.

TechmeIer, M. (1969) Music in the Teaching of French. Modern Language Journal, 53, 96.

Thain, L. (2010) Rhythm, Music and young Learners: A Winning Combination, en STOKE, A. M. (ed.) JALT 2009 Conference proceedings. Tokyo, 407-410.

Turnipseed, J. P. (1976) The Effect of Participation in Structured Classical Music Education Program on the Total Development of First Grade Children. Paper presented at the MidSouth Educational Research Conference. Kansas City.

TOSCANO-FuENTES, C. (2011) Estudio Empírico de la Relación existente entre el Nivel de Adquisición de una Segunda Lengua, la Capacidad Auditiva y la Inteligencia Musical del alumnado. Huelva, Universidad de Huelva.

TOURiÑÁn LóPEZ, J. M. y Longueira Matos, S. (2010) La música como ámbito de educación. Educación "por" la música y educación "para" la música. Teoría de la Educación. Revista Interuniversitaria, 22 (2), 151-181. 